\section{EFFICACY, DAMAGE ACCRUAL AND PREDICTORS OF RESPONSE TO BELIMUMAB IN ACTIVE SLE PATIENTS: A LARGE ITALIAN MULTICENTER PROSPECTIVE STUDY}

${ }^{1} \mathrm{M}$ Larosa* ${ }^{1} \mathrm{~L}$ laccarino, ${ }^{1} \mathrm{M}$ Zen, ${ }^{1} \mathrm{M}$ Gatto, ${ }^{2} \mathrm{~L}$ Emmi, ${ }^{3} \mathrm{~F}$ Conti, ${ }^{4} \mathrm{M}$ Mosca, ${ }^{5} \mathrm{P} . \mathrm{L}$. Meroni, ${ }^{6} \mathrm{M}$ Govoni, ${ }^{7} \mathrm{~S}$ De Vita, ${ }^{8} \mathrm{R}$ De Angelis, ${ }^{9} \mathrm{C}$ Salvarani, ${ }^{10} \mathrm{R}$ Gerli, ${ }^{11} \mathrm{~A}$ Tincani, ${ }^{1} \mathrm{~A}$ Doria. 1 Università degli Studi di Padova - Azienda Ospedaliera, Department of Medicine-DIMEDDivision of Rheumatology, Padova, Italy; ${ }^{2}$ University of Florence, Interdisciplinary Internal Medicine- Centre for Autoimmune Systemic Diseases- Behçet Centre and Lupus Clinic, Florence, Italy; ${ }^{3}$ Sapienza University, Rheumatology Unit- Department of Internal Medicine and Medical Specialities, Rome, Italy; ${ }^{4}$ University of Pisa, Department of Clinical and Experimental Medicine- Rheumatology Unit, Pisa, Italy; ${ }^{5}$ IRCCS Istituto- Auxologico Italiano, Experimental Laboratory of Immunological and Rheumatologic Researches, Milan, Italy; ${ }^{6}$ University of Ferrara, Rheumatology Unit- Department of Medical Sciences, Ferrara, Italy; "University Hospital "Santa Maria della Misericordia", Clinic of Rheumatology- DSMB, Udine, Italy; ${ }^{8}$ University of Jesi, Rheumatology Department, Jesi, Italy; ${ }^{9}$ Azienda OspedalieraIRCCS di Reggio Emilia, Rheumatology Unit- Department of Internal Medicine, Reggio Emilia, Italy; ${ }^{10}$ University of Perugia, Rheumatology Unit- Department of Medicine, Perugia, Italy; ${ }^{11}$ ASST Spedali Civili- University of Brescia, Rheumatology and Clinical Immunology Unit, Brescia, Italy

\subsection{6/lupus-2017-000215.95}

Background and aims To investigate effectiveness, damage accrual and predictors of response to belimumab in active SLE patients in clinical practice setting.

Methods 188 active SLE patients with anti-dsDNA antibodies and low C3 and/or C4, from 11 Italian centres, were treated with belimumab as add-on-therapy. Gender, age, disease duration, polyarthritis, skin rashes, glomerulonephritis, haematological involvement, SLEDAI- $\mathrm{K} \geq 10$, prednisone $\geq 7,5 \mathrm{mg} /$ day and concomitant immunosuppressants were used to determine baseline predictors of 12 - and 24 month response according to SRI-4. Data were analysed by SPSS (version 22.0).

Results Prominent clinical manifestations are summarised in Table 1. Clinical and serological variables at baseline, 12 and 24 months are reported in Table 2. SRI-4 was achieved by $71.3 \%$ and $68.7 \%$ of patients at 12 and 24 months, respectively. $92 \%$ of 12 month responders maintained SRI-4 response at 24 months; conversely, $87.5 \%$ of non-responders at 12 months were non-responders even at 24 months. Drug discontinuation for any cause was observed in $36.2 \%$ of patients; median treatment duration was 12 months. Damage accrual variation was significant between 5 years before drug initiation and baseline $(p<0.001)$, but not between baseline and the end of follow-up $(p=0.083)$. Baseline predictors of 12 month response were SLEDAI-2K $\geq 10$ (OR 25.8, 4.19159.2) and polyarthritis (OR 8.33, 1.88-36.78); 24 month response predictors were SLEDAI- $2 \mathrm{~K} \geq 10$ (OR 12.11, 1.6389.80 ), polyarthritis (OR 32.56, 2.94-360.56), and prednisone dose $\geq 7.5 \mathrm{mg} /$ day (OR 7.88, 1.02-61.48).

\begin{tabular}{|c|c|}
\hline Muscoloskeletal, number of patients (\%) & $85(45.2)$ \\
\hline Cutaneous, number of patients (\%) & $48(25.5)$ \\
\hline Haematological, number of patients (\%) & $26(13.8)$ \\
\hline Renal, number of patients (\%) & $24(12.8)$ \\
\hline Consitutional, number of patients (\%) & $3(1.6)$ \\
\hline Serositic, number of patients (\%) & $2(1.1)$ \\
\hline
\end{tabular}

\begin{tabular}{|c|c|c|c|c|}
\hline & Baseline & 12 months & 24 months & $\mathbf{p}$ \\
\hline SLEDAI-2K & $8.3 \pm 3.3$ & $4.2 \pm 2.7$ & $4.0 \pm 2.8$ & $<0.001$ \\
\hline Prednisone daily dose (mg/day) & $11.1 \pm 7.6$ & $4.9 \pm 2.9$ & $4.2 \pm 3.8$ & $<0.001$ \\
\hline DAS-28 score & $4.2 \pm 1.1$ & $2.2 \pm 1.0$ & $1.8 \pm 1.0$ & $<0.001$ \\
\hline CLASI activity score & $5.5 \pm 4.4$ & $1.4 \pm 2.9$ & $1.8 \pm 2.8$ & $<0.001$ \\
\hline 24-h proteinuria (g/die) & $1.13 \pm 0.73$ & $0.63 \pm 0.54$ & $0.54 \pm 0.60$ & 0.002 \\
\hline
\end{tabular}

Conclusions Belimumab seems to be effective and to reduce damage accrual in SLE patients in clinical practice setting. Patients with arthritis and high disease activity were the best responders to belimumab.

\section{URINARY BIOMARKER RELATED WITH TUBULAR INFLAMMATION IN LUPUS NEPHRITIS}

${ }^{1} \mathrm{EJ}$ Lee, ${ }^{1} \mathrm{~S}$ Hong, ${ }^{2} \mathrm{EJ}$ Chang, ${ }^{1} \mathrm{~B}$ Yoo, ${ }^{1} \mathrm{CK}$ Lee, ${ }^{1} \mathrm{YG}$ Kim. ${ }^{1}$ University of Ulsan College of Medicine- Asan Medical Centre, Department of Rheumatology, Seoul, Republic of Korea; ${ }^{2}$ University of Ulsan College of Medicine- Asan Medical Centre, Department of Biomedical Sciences-Cell Dysfunction Research Centre and BMIT, Seoul, Republic of Korea

\subsection{6/lupus-2017-000215.96}

Background and aims Severe tubulointerstitial damage represents a poor prognosis of lupus nephritis (LN). Immunoglobulin binding protein 1 (IGBP1) was known as a phosphoprotein associated with the immunoglobulin receptor in B cells. Our study determined urinary IGBP1 levels in LN patients and addressed correlations between urinary IGBP1 levels and clinical variables.

Methods The levels of IGBP1 were measured in urine of SLE patients with or without nephritis, and healthy subjects. Correlation analyses between urinary IGBP1 levels and the diseaserelated variables including C3/4, anti-dsDNA, and SLE Disease Activity Index (SLEDAI) were examined. We performed correlation analyses of urinary IGBP1 levels with activity index score or chronicity index score in renal tissues. To define IGBP1 expression in renal pathologies, immunohistochemical staining were performed. In addition, to identify genetic relationship with IGBP1, microarray was performed in HK2 tubular cells treated with IGBP1-siRNA.

Results Urinary levels of IGBP1 were significantly higher in LN patients than in SLE patients without nephritis and healthy individuals. Among the disease-related variables, SLEDAI scores, anti-dsDNA, and C3 were correlated with the levels of urinary IGBP1. Further, urinary IGBP1 levels represented a positive association with activity index score in renal pathology. IGBP1 was stained mainly in tubules, especially in class III, IV and V. In microarray analysis, IL-7 receptor was closely related with IGBP1 down-regulation.

Conclusions This study demonstrates that the levels of urinary IGBP1 were higher in LN patients and correlated with disease activity and activity index score of renal pathology. Therefore, urinary IGBP1 might be a valuable marker for determining the activity of LN. 